\title{
Phase diagram of a tetrahedral patchy particle model for different interaction ranges
}

\author{
Flavio Romano, ${ }^{1, a)}$ Eduardo Sanz, ${ }^{2}$ and Francesco Sciortino ${ }^{3}$ \\ ${ }_{1}^{1}$ Dipartimento di Fisica, Università di Roma La Sapienza, Piazzale A. Moro 2, 00185 Roma, Italy \\ ${ }^{2}$ SUPA, School of Physics and Astronomy, University of Edimburgh, Mayfield Road, Edinburgh EH9 3JZ, \\ United Kingdom \\ ${ }^{3}$ Dipartimento di Fisica and CNR-ISC, Università di Roma La Sapienza, Piazzale A. Moro 2, 00185 Roma, \\ Italy
}

(Received 4 February 2010; accepted 23 March 2010; published online 10 May 2010)

\begin{abstract}
We evaluate the phase diagram of the Kern-Frenkel patchy model with four interaction sites for four different values of the radial interaction range (all in the single-bond-per-patch regime) keeping the area of the interaction patches fixed. Four stable crystal phases are investigated, namely diamond cubic (DC), bcc, fcc, and plastic fcc. The DC is favored at low temperatures and pressures, while the bcc is favored at low temperatures and intermediate to high pressures. At low temperatures and very high pressures an ordered fcc phase is found, while - as expected — at high temperatures, the only stable crystal is a plastic fcc phase. We find a rich phase diagram with several re-entrant coexistence lines, which can be brought in the equilibrium phase diagram by a proper choice of the range. We also show that the gas-liquid phase separation becomes metastable as the range narrows, and it takes place in a region of the phase diagram where the low density diamond crystal is the thermodynamically stable phase. (C) 2010 American Institute of Physics. [doi:10.1063/1.3393777]
\end{abstract}

\section{INTRODUCTION}

Both for fundamental and technological reasons, particles with short-range, highly anisotropic (i.e., patchy) interactions have recently drawn a great deal of attention. The renewed interest in the study of patchy potentials, started two decades ago ${ }^{1,2}$ in the field of associated liquids, has been motivated by the possibility of synthesizing patchy particles that may self-assemble into new materials with potential technological applications. ${ }^{3,4}$ A lot of effort has been put, for example, in the crystallization of the diamond cubic crystal, a structure that is predicted to have photonic properties. ${ }^{5}$ It is therefore very valuable to deeply understand how the arrangement and nature of the interacting patches influences the thermodynamic stability of the target structures. Another topic where the physics of patchy particles might be relevant is the long-standing problem of protein crystallization, essential for the x-ray characterization of their structure. In a rough approximation, proteins can be regarded as patchy particles since their interactions are short-ranged and highly directional. ${ }^{6-8}$ The study of patchy interactions can therefore be helpful in understanding the mechanisms of protein crystallization.

Previous studies have revealed interesting differences in equilibrium and dynamic properties of patchy particles with respect to isotropic potentials. The gas-liquid separation, for example, moves toward low densities and temperatures ${ }^{9,10}$ as the number of patches per particle decreases, thus leaving behind a region of intermediate densities in which homogeneous gels can be formed. One result that carries over from isotropic to patchy interactions is the metastability of the

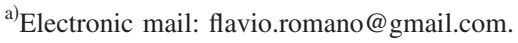

gas-liquid critical point with respect to crystallization for short interaction ranges. Furthermore, the extended law of corresponding states $^{11}$ has been shown to apply also for short-ranged patchy potentials ${ }^{12}$ among models with the same valence.

A full picture of the phase diagram of patchy potentials is already available for particles with octahedral, ${ }^{13,14}$ waterlike, ${ }^{2}$ and fcc-like ${ }^{14}$ symmetry, showing a rich variety of crystal phases, but the effect of the range has not been discussed in detail. We have recently studied, for a tetrahedral patchy model, the competition between crystallization and gas-liquid phase separation, finding that lowering the interaction range favors crystallization to a minor extent than it does for isotropic potentials. ${ }^{15}$ In this article, we present a complete thermodynamic study of the same model for several values of the interaction range, analyzing how the relative stability between the fluid and the crystal structures is modified by the bonding distance. This will provide some guidance in finding the optimal interaction parameters for the crystallization of the stable solid phases. Specifically, we quantify the region of stability of the technologically relevant DC structure and investigate how its melting temperature varies with the interaction range. The presented equilibrium phase diagram will serve as a starting point for studies of crystal nucleation in tetrahedral liquids. ${ }^{16-19}$

\section{THE MODEL}

We study the tetravalent Kern-Frenkel ${ }^{20}$ model for patchy particles, with four directionally attractive sites on the surface of a hard sphere located in a tetrahedral fashion. The 


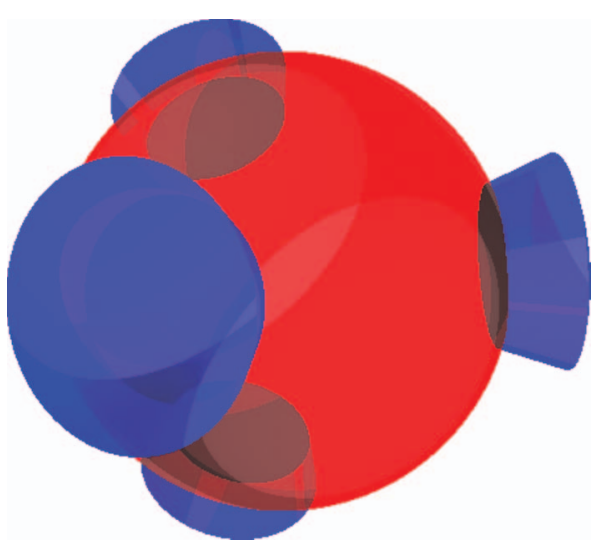

FIG. 1. Graphical representation of a particle as described by the KernFrenkel model. The four interaction sites are arranged on the vertexes of a regular tetrahedron inscripted in a impenetrable sphere of diameter $\sigma$. The patches' attractive regions are represented by the dark sections of spherical cap of height $\Delta$ and aperture $\pm \theta_{\max }$.

two-body potential is a square-well potential modulated by a function that depends only on the relative orientation of the interaction sites (patches) on the particles,

$$
u\left(\mathbf{r}_{i j}\right)=u_{\mathrm{SW}}\left(r_{i j}\right) f\left(\left\{\Omega_{i}, \Omega_{j}\right\}\right) .
$$

The prefactor $u_{\mathrm{SW}}\left(r_{i j}\right)$ is a well potential of attractive range $\Delta$ and depth $u_{0}$, while the modulating function $f$ is defined as follows; let $\hat{\mathbf{u}}_{i}^{\alpha}$ be the normalized vector that, starting from the center of the particle $i$, points toward the interaction site labeled by $\alpha$ (ranging from 1 to 4 ) on its surface, and let $\hat{\mathbf{r}}_{i j}$ be the normalized vector connecting the centers of particles $i$ and $j$. The function $f$ reads

$$
f\left(\Omega_{i}, \Omega_{j}\right)=\left\{\begin{array}{c}
-1 \text { if }\left\{\begin{array}{l}
\hat{\mathbf{r}}_{i j} \cdot \hat{\mathbf{u}}_{i}^{\alpha}>\cos \left(\theta_{\max }\right) \text { for any } \alpha, \\
\text { and } \\
\hat{\mathbf{r}}_{j i} \cdot \hat{\mathbf{u}}_{j}^{\alpha}>\cos \left(\theta_{\max }\right) \text { for any } \alpha,
\end{array}\right. \\
0 \text { otherwise. }
\end{array}\right.
$$

Then, to form a bond, two particles must be within the square well range and each particle must have a patch that forms an angle less than a maximum angle $\theta_{\max }$ with the vector connecting the centers of the two particles. A pictorial representation of a such particle is given in Fig. 1. We stress that in this model the angular $\left(\theta_{\max }\right)$ and radial $(\Delta)$ interaction ranges are decoupled, giving the possibility of changing the maximum bonding distance keeping fixed the fraction of the particle surface covered by interaction patches $\chi=4[(1$ $\left.\left.-\cos \left(\theta_{\max }\right)\right) / 2\right]$.

We study the thermodynamic behavior of this model at fixed $\theta_{\max }=\operatorname{acos}(0.92)$ for four different values of the sitesite interaction range $\Delta / \sigma=\delta=0.03,0.07,0.12$, and $0.24, \sigma$ being the particle's diameter. All these parameters respect the single-bond-per-patch condition, which is ensured for geometrical reasons ${ }^{12}$ if $\sin \left(\theta_{\max }\right) \leq 1 /(2(1+\delta))$. Throughout all this work, we use reduced units with $\sigma=1$ and $u_{0}=1$, temperature $T$ is measured in units of $u_{0} / k_{\mathrm{B}}$, number density $\rho$ in units of $\sigma^{-3}$, and pressure $P$ in units of $u_{0} / \sigma^{3}$.

\section{FREE ENERGY CALCULATIONS}

An extensive review of all the techniques needed for the computation of phase diagrams via numerical simulation has been recently published by Vega et al. ${ }^{21}$ Both free energy computation and coexistence lines tracing methods are covered, and all the work presented in this article follows the guidelines in Ref. 21.

Computing a phase diagram entails finding the points in the $P-T$ plane for which two phases have the same chemical potential $\mu=A / N+p / \rho$. We compute the Helmholtz free energy $A$ of a thermodynamic state point starting from a reference system with known free energy - either analytically or by numerical calculations. The free energy of the investigated system can then be recovered by integrating its free energy difference with respect to the reference system along a (non-necessarily physically meaningful) path connecting the two. While conceptually identical, the procedure is technically different for fluid and crystal phases.

\section{A. Fluid free energy}

The Helmholtz free energy $A$ of the gas and fluid phases at density $\rho$ and temperature $T$ is computed taking as a reference state the ideal gas at the same thermodynamic conditions. The final expression reads, in reduced units where $\Lambda_{\text {De Broglie }}=1,{ }^{21}$

$$
\frac{A^{\text {fluid }}(T, \rho)}{N k_{\mathrm{B}} T}=\frac{A^{\text {ideal gas }}(\rho)}{N k_{\mathrm{B}} T}+\int_{0}^{\rho}\left[\frac{p}{k_{\mathrm{B}} T \rho^{\prime 2}}-\frac{1}{\rho^{\prime}}\right] \mathrm{d} \rho^{\prime},
$$

with

$$
\frac{A^{\text {ideal gas }}}{N k_{\mathrm{B}} T}=\ln (\rho)-1+\frac{1}{N} \ln (2 \pi N),
$$

where we included $\mathcal{O}(1 / N)$ finite-size corrections to the ideal gas free energy. Note that the contribution to the entropy of the ideal gas arising from the orientational degrees of freedom is not accounted for here and will be taken into account as normalization into the orientational part of the crystals' free energy, as discussed below.

The most accurate procedure to compute the integral in Eq. (3) is achieved by running several NPT ensemble simulations and then integrating a polynomial fit of the integrand function. Having the latter a factor $\rho^{2}$ at the denominator, it is difficult to estimate at low densities, but as pointed out in Ref. 21 it is easy to show that its intercept is the second virial coefficient $B_{2}$. The analytic form for the Kern-Frenkel model $B_{2}$, reported in Ref. 20, reads

$$
\frac{B_{2}}{B_{2}^{\mathrm{HS}}}=1-\chi^{2}\left[(1+\delta)^{3}-1\right]\left(\mathrm{e}^{u_{0} / k_{\mathrm{B}} T}-1\right),
$$

where $B_{2}^{\mathrm{HS}}=(2 / 3) \pi \sigma^{3}$ is the hard spheres' second virial coefficient and $\chi$ is defined in Sec. II.

The right hand side of Eq. (3) was computed by means of isotropic NPT Monte Carlo (MC) simulations on systems of 250 particles. Simulations at the highest $\delta$ were run for at least $10^{5} \mathrm{MC}$ steps (each defined as $N$ translational attempts, $N$ rotation attempts, and 1 volume change attempt), one third of which were used for equilibration. Low- $\delta$ runs required 


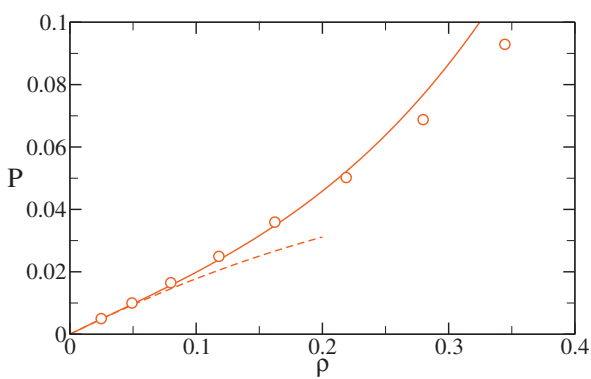

FIG. 2. Fluid equation of state at $\delta=0.12$ and $T=0.2$ (i.e., close to $T_{\mathrm{c}}$ $=0.1573$ ). Numerical results (open circles) compared with WTPT (solid line) and the virial expansion up to $B_{2}$ (dashed line). The WTPT follows the numerical results up to density of the order of 0.2 .

much longer equilibration and sampling times, at least $10^{6}$ MC steps. At least ten pressures were sampled at each temperature.

Note that, in the case of patchy particles, a reasonable theoretical expression for the fluid equation of state can be formulated implementing the thermodynamic perturbation Wertheim theory (WTPT). ${ }^{22,23}$ Such theory has been successfully used for reproducing the low density behavior of models for associating fluids such as the one treated here. ${ }^{24-26} \mathrm{~A}$ comparison between the numerical and theoretical equation of state for the present model is shown in Fig. 2.

\section{B. Crystals' free energy}

The free energy of the solid phases are computed taking as a reference model an ideal Einstein crystal with fixed center of mass ${ }^{27}$ and tetrahedral symmetry ${ }^{21}$ with Hamiltonian

$$
\begin{aligned}
H_{\text {Einst }}\left(\lambda_{\text {tras }}, \lambda_{\text {or }}\right)= & \lambda_{\text {tras }} \sum_{i=0}^{N}\left(\mathbf{r}_{i}-\mathbf{r}_{i, 0}\right)^{2} \\
& +\lambda_{\text {or }} \sum_{i=0}^{N}\left(\sin ^{2}\left(\psi_{i \mathrm{a}}\right)+\sin ^{2}\left(\psi_{i \mathrm{~b}}\right)\right),
\end{aligned}
$$

where the term $\left(\mathbf{r}_{i}-\mathbf{r}_{i, 0}\right)$ is the displacement of the particle $i$ from its rest position in the ideal Einstein crystal and the angles $\psi_{i \mathrm{a}}$ and $\psi_{i \mathrm{~b}}$ are the minimum angles formed by the vector defining any of the patches of particle $i$ and the rest position of the patch a and $\mathrm{b}$ respectively, provided that $\psi_{i \mathrm{a}}$ and $\psi_{i \mathrm{~b}}$ are not formed by the same patch in the ideal Einstein crystal. $\lambda_{\text {tras }}$ and $\lambda_{\text {or }}$ are the translational and orientational coupling parameters respectively. The issue of the fixed center of mass is discussed in detail in Refs. 28 and 21. We stress that the Hamiltonian in Eq. (6) has the same symmetry of the chosen Kern-Frenkel model. The Hamiltonian in Eq. (6) leads to the following expression for the reference free energy, split in the orientational and translational contributions,

$$
A_{\text {Einst }}^{(C M)}=A_{\text {Einst-tras }}^{(C M)}+A_{\text {Einst-or }}
$$

where we defined

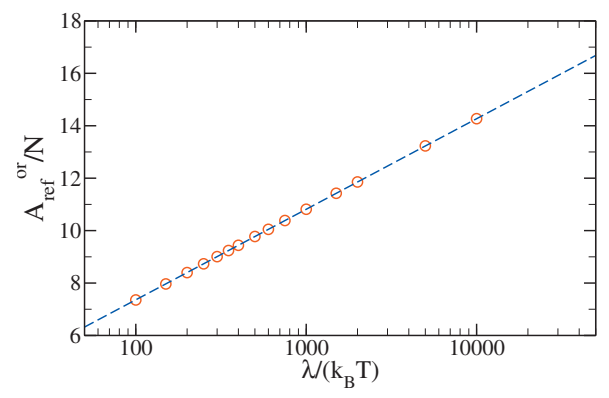

FIG. 3. Orientational reference free energy per particle versus the orientational coupling parameter $\lambda_{\text {or }}$ divided by $k_{\mathrm{B}} T$. The circles are the points computed with a MC integration algorithm and the dashed line is a fit to a function $A_{\text {Einst }}^{\text {or }}\left(T, \lambda_{\text {or }}\right) / N=a+(3 / 2) \ln \left(\lambda_{\text {or }} /\left(k_{\mathrm{B}} T\right)\right)$. The resulting best fit function, with $a=0.4524 \pm 0.0005$, was used for the calculations presented in Sec. VI. Note the log scale on the $x$-axis.

$$
\frac{A_{\text {Einst-tras }}^{(C M)}}{N k_{\mathrm{B}} T}=-\frac{3}{2} \frac{N-1}{N} \ln \left(\frac{\pi k_{\mathrm{B}} T}{\lambda_{\text {tras }}}\right)-\frac{3}{2 N} \ln (N),
$$

as the translational free energy of the ideal Einstein crystal with fixed center of mass (including $O(\ln (N))$ terms), while

$$
\begin{aligned}
\frac{A_{\text {Einst-or }}^{(C M)}}{N k_{\mathrm{B}} T}= & -\ln \left\{\frac{1}{8 \pi^{2}} \int \mathrm{d} \theta \sin (\theta) \mathrm{d} \phi \mathrm{d} \chi\right. \\
& \left.\times \exp \left[-\frac{\lambda_{\text {or }}}{k_{\mathrm{B}} T}\left(\sin ^{2}\left(\psi_{i \mathrm{a}}\right)+\sin ^{2}\left(\psi_{i \mathrm{~b}}\right)\right)\right]\right\}
\end{aligned}
$$

is the orientational free energy of the ideal Einstein crystal $(\theta, \phi$, and $\chi$ are the Euler angles). The solid angle normalization factor $1 /\left(8 \pi^{2}\right)$ is consistent with the choice of Eq. (4) for the ideal gas free energy.

The integral in Eq. (9) is the one-particle angular partition function of the reference Einstein crystal and, in our case, has to be computed by numerical means. To this extent, we used a MC integration with at least $10^{9}$ function calls for each calculation. As reported in Fig. 3, we found that the values of $A_{\text {Einst }}^{\text {or }}$ as a function of $\lambda_{\text {or }} /\left(k_{\mathrm{B}} T\right)$ are well described by a logarithmic fit over several orders of magnitude, so we used the fitting function to compute $A_{\text {Einst-or }}$ for the calculations reported below.

Once the free energy of the reference crystal is known, one can compute the free energy of the investigated crystal with the following formula:

$$
A=A_{\text {Einst }}^{(C M)}+\Delta A_{1}+\Delta A_{2}+\Delta A_{3} .
$$

Following Ref. 21, we define $\Delta A_{1}$ as the free energy difference between the ideal Einstein crystal with fixed center of mass and the interacting Einstein crystal with fixed center of mass, $\Delta A_{2}$ as the free energy difference between the interacting Einstein crystal with fixed center of mass and the investigated crystal with fixed center of mass, and $\Delta A_{3}$ as the free energy difference between a solid with fixed center of mass and a solid with unconstrained center of mass. We used the following standard expressions for the terms $\Delta A_{1}$ and $\Delta A_{3}$,

$$
\begin{aligned}
\frac{\Delta A_{1}}{N k_{\mathrm{B}} T}= & -\frac{1}{N} \ln \left\langle\exp \left\{-\left(\frac{U_{\text {sol }}\left(\mathbf{r}_{i}, \mathbf{r}_{j}\right)}{k_{\mathrm{B}} T}-\frac{U_{0}}{k_{\mathrm{B}} T}\right)\right\}\right\rangle_{\text {Einst-id }} \\
& +\frac{U_{0}}{N k_{\mathrm{B}} T}
\end{aligned}
$$


TABLE I. Free energies as computed with the Frenkel-Ladd procedure. Free energies are in units of $k_{\mathrm{B}} T$ per particle. All symbols are defined in the text.

\begin{tabular}{lccccccrrrrr}
\hline \hline Phase & $\delta$ & $N$ & $T$ & $\rho$ & $\Lambda_{\text {tras }} \sigma^{2} /\left(k_{\mathrm{B}} T\right)$ & $\eta /\left(k_{\mathrm{B}} T\right)$ & $A_{\text {Einst }}^{\text {or }}$ & $\Delta A_{1}$ & $\Delta A_{2}$ & $\Delta A_{3}$ & $A$ \\
\hline DC & 0.07 & 216 & 0.15 & 0.6045 & 10000 & 1.00 & 17.113 & -13.333 & -12.474 & 0.001 \\
bcc & 0.07 & 250 & 0.15 & 1.1586 & 6000 & 0.01 & 9.440 & -13.333 & -12.169 & 0.001 & -1.974 \\
DC & 0.12 & 216 & 0.10 & 0.5600 & 7500 & 1.00 & 17.290 & -20.000 & -23.217 & 0.001 & -10.912 \\
bcc & 0.12 & 250 & 0.20 & 1.1500 & 5000 & 1.00 & 15.642 & -10.000 & -18.527 & 0.001 & 0.502 \\
\hline \hline
\end{tabular}

$$
\frac{\Delta A_{3}}{N k_{\mathrm{B}} T}=-\frac{1}{N} \ln (\rho) .
$$

The average in Eq. (11) is taken sampling with the Einstein Hamiltonian alone. Here $U_{\text {sol }}$ is the potential energy of the Kern-Frenkel (in our case) model as sampled in the simulation, while $U_{0}$ is the Kern-Frenkel potential energy of the reference Einstein crystal, and is thus fixed. Our choice of $\Delta A_{3}$ is due to the fact that we performed simulations of the interacting Einstein crystal with fixed center of mass. ${ }^{21}$

The heart of the free energy calculation is the free energy difference between the investigated crystal and the interacting Einstein crystal, both with fixed center of mass, $\Delta A_{2}$. For numerical reasons, we do not choose the two coupling parameters of the Einstein Hamiltonian Eq. (6) to be equal, having found that a better precision could be achieved if we introduced a multiplicative factor in between them, that is

$$
\lambda=\lambda_{\text {tras }}=\lambda_{\mathrm{or}} / \eta,
$$

this leads to the following form for $\Delta A_{2}$ :

$$
\begin{aligned}
\Delta A_{2} & \equiv-\int_{0}^{\lambda_{\max }} \mathrm{d} \lambda\left\langle\frac{\partial H_{\text {Einst }}(\lambda, \eta)}{\partial \lambda}\right\rangle_{\lambda} \\
& =-\int_{0}^{\lambda_{\max }} \mathrm{d} \lambda\left\langle\frac{\partial H_{\text {tras }}^{\text {Einst }}(\lambda)}{\partial \lambda}+\eta \frac{\partial H_{\text {or }}^{\text {Einst }}(\lambda)}{\partial \lambda}\right\rangle_{\lambda} .
\end{aligned}
$$

Here, $\lambda_{\max }$ is chosen in such a way that the system behavior is completely determined by the reference Einstein Hamiltonian. The choice (13) is due to an imbalance between the field needed to avoid hard-core overlaps and the one needed to avoid that a particle breaks its bonds by rotating. On lowering $\delta$, one has to increase $\lambda_{\max }$ to account for the increased density of the crystals, while the orientational field does not need to be increased. The introduction of the factor $\eta$ allowed us to independently choose the orientational and translational contributions without increasing the number of simulations needed to compute $\Delta A_{2}$. The integrals in Eq. (14) were computed running 10-20 fixed- $N V T \lambda$ MC simulations of the interacting Einstein crystal with fixed center of mass at $\log$-spaced values of $\lambda$ for at least $10^{5} \mathrm{MC}$ steps as defined in Sec. III. The values of $\lambda_{\text {tras }}$ and $\eta$ were chosen so that $\Delta A_{1}$ $=-2 N u_{0}$ [i.e., the average in Eq. (11) is equal to one] over a $10^{5}$ steps long $\mathrm{MC}$ simulation of the noninteracting Einstein crystal. A few examples of the free energies thus obtained are reported in Table I.

The form of Eqs. (10), (11), and (14) suggests that when the temperature is so low that all possible bonds are formed and the energy remains fixed to the ground state value throughout all the integration procedure, one can separately compute the free energy contributions associated to the ori- entational and translational degrees of freedom. Hence, we define the translational and orientational entropy contributions $S_{\text {tr }}$ and $S_{\text {or }}$, as

$$
\begin{aligned}
& \frac{S_{\mathrm{tr}}}{N k_{\mathrm{B}}}=-\frac{A_{\text {Einst-tras }}^{(C M)}}{N k_{\mathrm{B}} T}+\int_{0}^{\lambda_{\max }} \mathrm{d} \lambda\left\langle\frac{\partial H_{\text {tras }}^{\text {Einst }}}{\partial \lambda}\right\rangle-\Delta A_{3}, \\
& \frac{S_{\text {or }}}{N k_{\mathrm{B}}}=-\frac{A_{\text {Einst-or }}^{(C M)}}{N k_{\mathrm{B}} T}+\int_{0}^{\lambda_{\max }} \mathrm{d} \lambda\left\langle\eta \frac{\partial H_{\text {or }}^{\text {Einst }}}{\partial \lambda}\right\rangle .
\end{aligned}
$$

\section{COEXISTENCE LINES}

\section{A. Gas-liquid}

The gas-liquid coexistence lines were computed via the Gibbs ensemble simulation technique. ${ }^{29}$ A system of 216 particles is divided in two boxes of total volume $1100 \sigma^{3}$. The top of our coexistence is completed with the critical parameters of Ref. 12 at $\delta=0.03,0.07$, and 0.12 , while the critical point at $\delta=0.24$ has been calculated here as described in Ref. 12. The coexistence pressures between the gas and liquid phases were computed via a bracketing procedure with the aid of fixed-NPT MC simulations. Close to the critical temperature, a pressure slightly lower than the critical value pushes the system toward low densities (gas phase), while a pressure slightly higher pushes the system toward high density (liquid phase). Knowing the critical temperatures and densities from Ref. 12, at each $\delta$ studied, we ran several simulations at the critical temperature bracketing the critical pressure.

\section{B. Fluid-crystal and crystal-crystal}

Once the free energy of a state point is known, one has to integrate the free energy difference at fixed temperature (or pressure) to obtain the chemical potential $\mu=A / N+P / \rho$ as a function of the pressure (or temperature). One then defines the coexistence temperature and pressure to satisfy

$$
\mu_{\text {phase I }}\left(P_{\text {coex }}, T_{\text {coex }}\right)=\mu_{\text {phase II }}\left(P_{\text {coex }}, T_{\text {coex }}\right) \text {. }
$$

Starting from the found coexistence point in the $(T-P)$ plane, one can calculate the whole coexistence line by means of the Gibbs-Duhem integration technique introduced by Kofke, ${ }^{30}$ which consists in numerically integrating the Clausius-Clapeyron equation, expressing the variation of the pressure versus temperature along the coexistence $\left(\left.\right|_{\mathrm{X}}\right)$, as

$$
\left.\frac{d P}{d T}\right|_{\mathrm{x}}=\frac{\Delta U+P \Delta V}{T \Delta V},
$$

starting from the known coexistence point. In Eq. (18), $\Delta U$ and $\Delta V$ are the differences between the thermodynamic av- 
TABLE II. Fluid-crystal coexistence points checked with the direct coexistence technique by Ladd and Woodcock (Ref. 31). The errors represent the difference between the lowest temperature that led to crystallization with respect to the highest temperature that led to melting.

\begin{tabular}{lcccc}
\hline \hline Coexisting phases & $\delta$ & $p$ & Predicted $T_{\text {coex }}$ & Actual $T_{\text {coex }}$ \\
\hline DC-fluid & 0.03 & 0.300 & 0.1347 & $0.135 \pm 0.002$ \\
bcc-fluid & 0.03 & 0.392 & 0.1397 & $0.140 \pm 0.003$ \\
DC-fluid & 0.07 & 0.080 & 0.1530 & $0.153 \pm 0.002$ \\
bcc-fluid & 0.07 & 0.300 & 0.1636 & $0.163 \pm 0.001$ \\
\hline \hline
\end{tabular}

erages of the energy and volume of the two coexisting phases as obtained by numerical simulations. Depending on the slope $d P /\left.d T\right|_{\mathrm{x}}$, one can decide to integrate the inverse of Eq. (18) to achieve a better numerical precision or equivalently a faster estimate. All the coexistence lines between ordered and disordered phases were obtained with the Gibbs-Duhem technique, using a fourth order Runge-Kutta algorithm. The slope $d P / d T$ was obtained running separate $N P T$ simulations of the two phases. Simulations for the fluid phases were run on systems with $N=250$ particles for at least $10^{6} \mathrm{MC}$ steps, one third of which were used for equilibration. Simulations of the crystal phases were done with systems of $N=250,216$, and 256 for the bcc, DC, and fcc phase respectively, and were run for at least $10^{5} \mathrm{MC}$ steps.

\section{CONSISTENCY CHECKS}

As pointed out in Ref. 21, it is wise to check some of the free energies and coexistence points obtained with independent approaches. Several checks can be made for both kinds of computations. As far as the fluid is concerned, its free energy as computed by thermodynamic integration at a given temperature and density can be checked in a straightforward way by running a grand canonical MC simulation ${ }^{28}$ at the computed chemical potential to verify that the predicted equilibrium density is reached. We used this check once at every range studied obtaining consistent results.

A completely independent check useful for solid-fluid coexistence lines is the Ladd-Woodcock direct coexistence technique, ${ }^{31}$ consisting in running a simulation at the predicted coexistence temperature and pressure of a system composed of equilibrated configurations of the two coexisting phases. If the predicted coexistence point is correct, the two phases will stay at equilibrium indefinitely, while if the parameters are not correct one phase will prevail. We checked several coexistence points, either obtained directly from free energy calculations or by Gibbs-Duhem integration, with the direct coexistence technique. A few examples are reported in Table II. The comparison between the predicted coexistence temperatures and the temperatures found via direct simulations of the coexisting phases (last two columns of the table) confirm the stability of the methodology and its accuracy.

We used the Hamiltonian Gibbs-Duhem integration ${ }^{32}$ to compute a coexistence point at any $\delta$ starting from a known coexistence point at a given $\delta$. The basic idea of this tech- nique is to introduce a coupling parameter $\lambda$, ranging from 0 to 1 , that combines two potentials of the same symmetry as follows:

$$
u=(1-\lambda) u_{1}+\lambda u_{2} .
$$

It has been shown ${ }^{32}$ that one can obtain the following generalized Clapeyron equations in the $T-\lambda$ or $P-\lambda$ plane for two coexisting phases I and II,

$$
\begin{aligned}
& \left.\frac{d T}{d \lambda}\right|_{\mathrm{x}}=T \frac{\left\langle\partial u_{\mathrm{II}} / \partial \lambda\right\rangle_{N P T \lambda}-\left\langle\partial u_{\mathrm{I}} / \partial \lambda\right\rangle_{N P T \lambda}}{h_{\mathrm{II}}-h_{\mathrm{I}}}, \\
& \left.\frac{d P}{d \lambda}\right|_{\mathrm{x}}=-\frac{\left\langle\partial u_{\mathrm{II}} / \partial \lambda\right\rangle_{N P T \lambda}-\left\langle\partial u_{\mathrm{I}} / \partial \lambda\right\rangle_{N P T \lambda}}{v_{\mathrm{II}}-v_{\mathrm{I}}} .
\end{aligned}
$$

Lower case letters refer to thermodynamic variables per particles. $h$ is the enthalpy, $v$ is the specific volume, and $u$ is the configurational energy. If we choose 1 and 2 to be the same model at two different values of the interaction range $\delta$, we can compute the coexistence point at one value of $\delta$ starting from another $\delta$ at which the coexistence point is known by numerically integrating Eq. (20) or (21) from $\lambda=0$ to $\lambda=1$. This technique is not only useful for checks between the coexistence lines obtained at different $\delta$ 's, but can in principle be used to obtain the whole phase diagram of a model starting from the phase diagram of a different model. ${ }^{33} \mathrm{We}$ found that the Hamiltonian integration is faster than repeating the whole Frenkel-Ladd procedure, so we used Hamiltonian Gibbs-Duhem integration to obtain the phase diagram at the smallest range studied $\delta=0.03$ starting from $\delta=0.07$, and then checked some of the points obtained with the direct coexistence technique (see Sec. II).

\section{RESULTS}

In order to compute the phase diagram, we considered four crystalline phases: fcc, both orientationally ordered (fcc) and disordered (fcc-d), DC, and bcc. A pictorial representation of these structures is reported in Fig. 4. The fcc is the close packed structure with 12 nearest neighbors. ${ }^{34}$ Each particle is bonded to four nearest neighbors, which are arranged on four of the vertexes of a octahedron, a structure which does not completely reflect the symmetry of the model. Still, the finite width of the patch angle allows for full bonding also in the fcc structure. The DC crystal is the structure of Ice Ic ${ }^{35}$ with eight particles per unit cell and a close packing density $\rho_{\mathrm{DC}}^{\max }=3^{3 / 2} / 8=0.6459$. In this case, one particle has all of its four nearest neighbors arranged on a tetrahedron, thus reflecting its symmetry. The bcc crystal is the structure of Ice VII. ${ }^{35}$ It has two atoms per unit cell and a close packed density $\rho_{\mathrm{bcc}}^{\max }=3^{3 / 2} / 4=1.2989$. This crystal is made up of two interpenetrating DC lattices, so that each particle has eight nearest neighbors, four of which are bonded. The bcc and DC phases have nearest neighbors forming an exact tetrahedral angle $\theta_{\text {tetra }} \simeq 109^{\circ}$, while the fcc does not. At low $T$, all these crystals have a ground-state energy equal to $-2 N u_{0}$. We also study the stability field of the orientationally disordered (plastic) fcc-d phase. Indeed, as expected, this crystal will eventually become the only thermodynamically stable crystal at high $T$, since the Kern-Frenkel model reduces to that of 

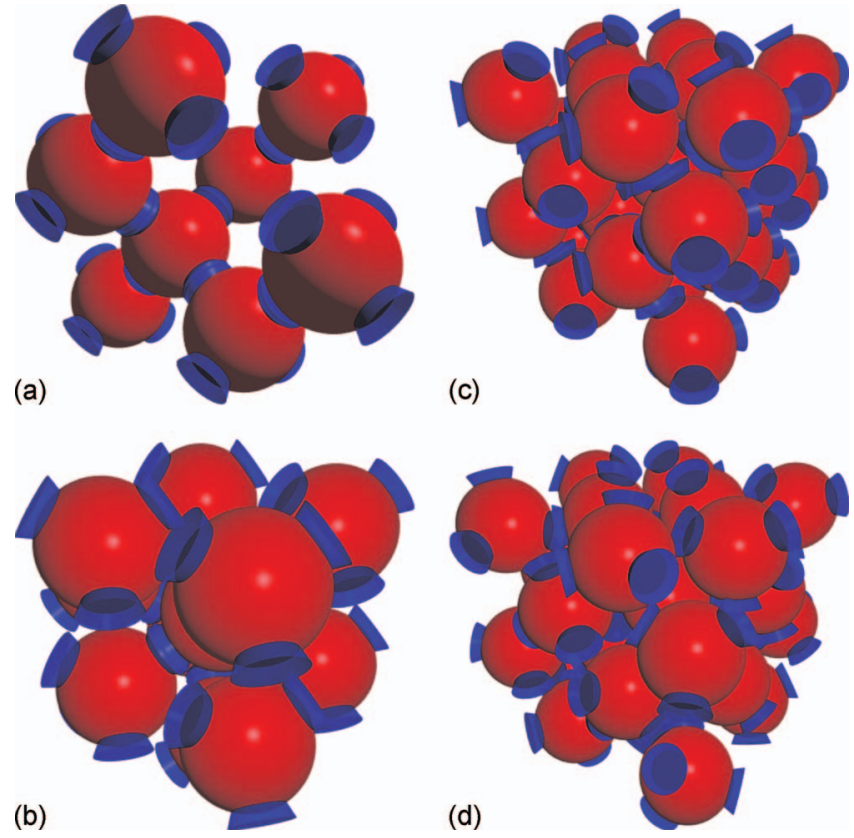

FIG. 4. Crystal structures present in the phase diagram; (a) DC (one unit cell, composed of eight atoms); (b) bcc (eight atoms in the vertices of a cube around the central one); (c) fcc $(2 \times 2 \times 2$ unit cells, composed of four atoms each); (d) fcc-d. $(2 \times 2 \times 2$ primitive cells, composed of four atoms each). Note the random orientations of the patches in (d) with respect to (c).

hard spheres. ${ }^{27}$ The transition at high $T$ to fcc-d has been observed in the study of other patchy models, in the limit where patches become irrelevant. ${ }^{13}$

The phase diagrams obtained are presented in Figs. 5 and 6. As discussed in detail in Ref. 15, the gas-liquid critical point becomes metastable when the range becomes smaller than $\delta \leq 0.15$. The liquid phase disappears from the equilibrium phase diagram, existing only as a metastable state. We have never observed spontaneous crystallization of the metastable liquid into the stable DC crystal, suggesting that the activation barriers for crystallization of the present model, for all $\delta$, are large and do not allow for spontaneous crystallization within the explored computational time. The evolution with $\delta$ of the critical parameters has been studied previously ${ }^{12}$ and it has been shown that while the critical density scales as $(1+\delta / 2)^{-3}$, the critical temperature is well approximated by a constant value of the reduced second virial coefficient $B_{2} / B_{2}^{\mathrm{HS}} \approx-4.5$.

A global look at Figs. 5 and 6 shows immediately that the two phases that appear to be more sensitive to the value of the range are the bcc and the fcc. Indeed, on increasing the range, the field of stability of both crystals expand. This is evident in the $T-\rho$ plane (Fig. 6), where the region of densities where the fcc and bcc phase are stable significantly grows. There is a limitation on the maximum value of $\delta$ ( 0.276 in the present model) where the single-bond-per-patch condition applies and the ground state energy is fully determined by the number of patches. In this scenario, all fully bonded structures share a common coexistence point at $T$ $=0$ and $P=0$, where neither density or entropy matter and they all have the same configurational energy. At zero pressure and small but positive temperature, the higher free volume available in the open structure DC results in a higher entropy (a quantitative discussion of this is given below) and thus dominates over bcc. In this respect, it is impossible to increase $\delta$ to values such that the bcc structure always dominates over the DC without altering the bonding pattern.

At equilibrium, at low densities and temperatures, the system phase separates in a gas coexisting with a DC crystal. This structure is the lowest density fully bonded crystalline structure for tetrahedral particles. The region of densities where the DC crystal is stable is very narrow. As the inter-
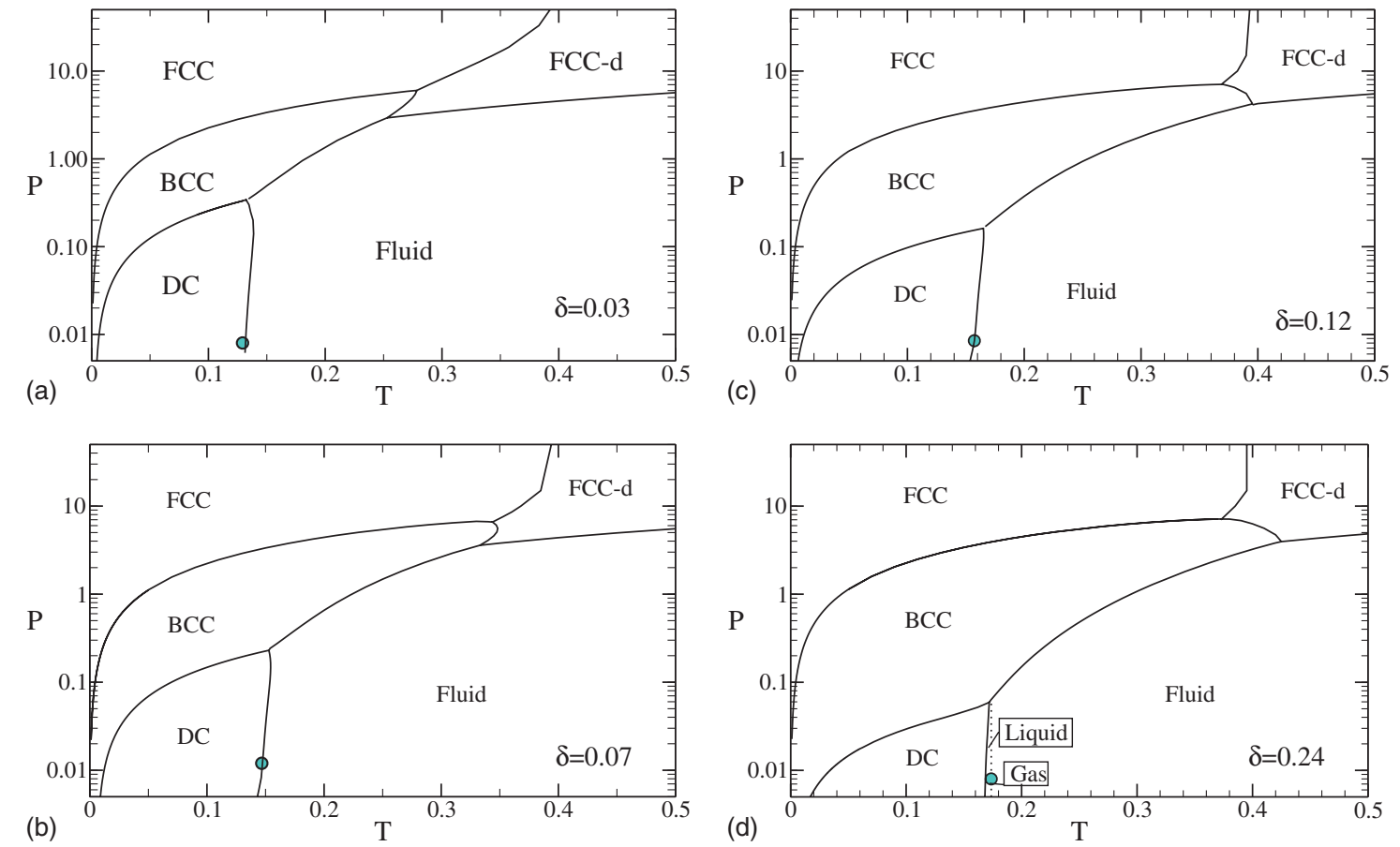

FIG. 5. Phase diagrams in the $P-T$ plane. We use reduced units defined in Sec. II. The gas-liquid critical temperatures are taken from Ref. 12, while the critical pressures have been computed as described in Sec. IV A. 

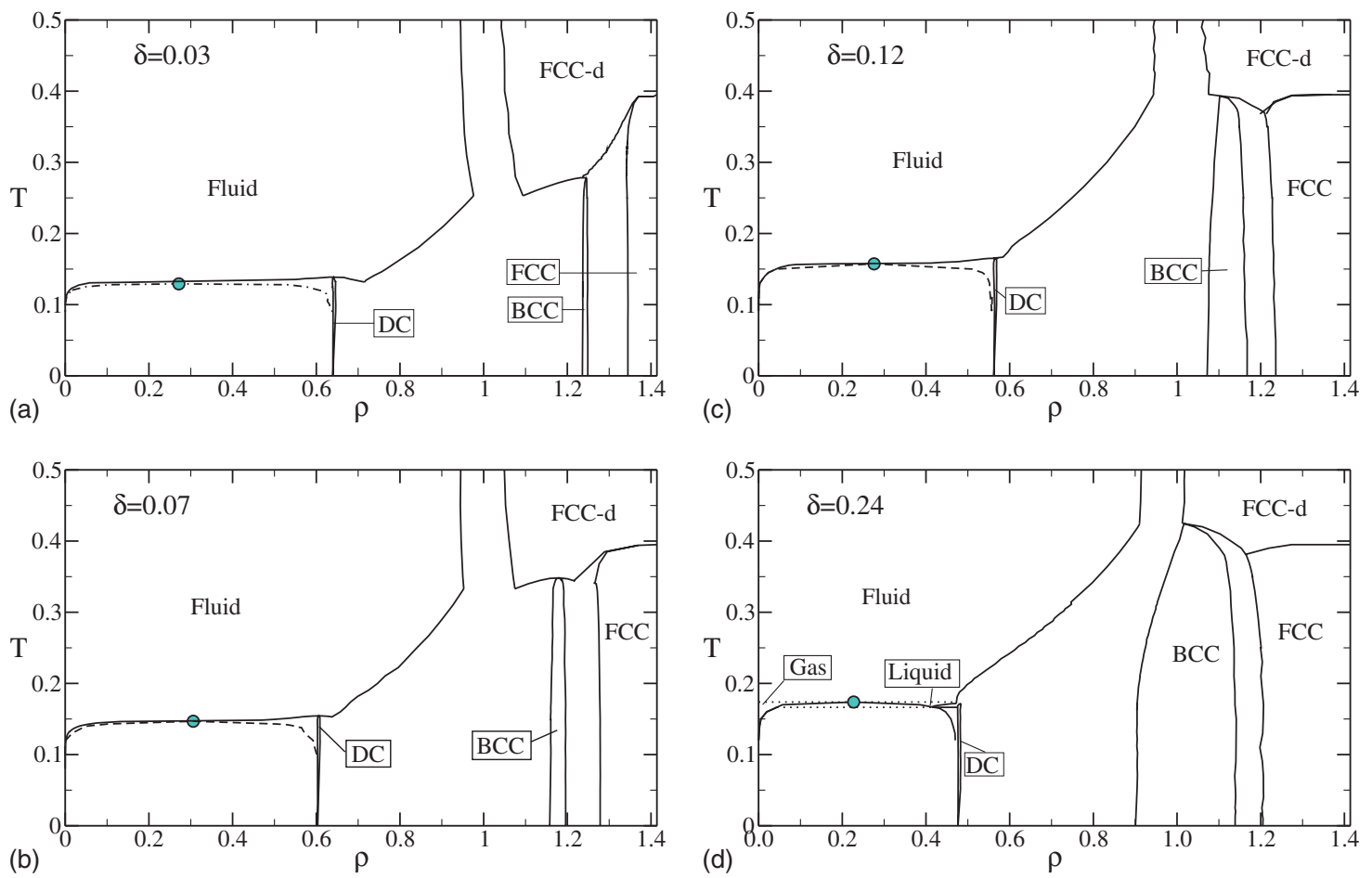

FIG. 6. Phase diagrams in the $\rho-T$ plane. We use reduced units as defined in Sec. II. The gas-liquid coexistence lines (dashed) are metastable with respect to crystallization to the DC phase at the three lowest values of the interaction range studied.

action range increases, the DC phase melts at higher temperatures, suggesting that crystallization of the DC phase could be facilitated by the choice of a large interaction range (but still within the single bond per patch condition).

The phase diagrams reported in Figs. 5 and 6 also show an interesting behavior concerning the possibility of a reentrant melting of the DC phase. Indeed the boundary between the DC and the fluid becomes negatively sloped (in the $(P-T)$ plane $)$ at high pressures, generating a nose which, for small values of $\delta$, can be accessed in equilibrium. Indeed (data not shown), at high pressures, the metastable extension of the DC-fluid coexistence inside the stable bcc region is always negatively sloped, as previously observed in Ref. 2 . This re-entrant melting appears to be a typical feature of open crystals. Re-entrant melting is a consequence of the fact that the compressibility of the fluid is much larger than that of the solids. Thus, by increasing the pressure, the fluid can become denser than open crystal structures, making the coexistence line's slope negative. ${ }^{36}$ The re-entrant phenomenon appears as a maximum of $T$ versus $\rho$ along coexistence at densities around $\rho \approx 0.6$. At the maximum, the fluid and DC crystals have the same equilibrium density.

Another re-entrant phase boundary is found for the bccfcc-d coexistence. On increasing pressure, the fcc-d transforms into bcc and then into fcc-d again. As in the DC-fluid case, the re-entrance can take place at equilibrium [case $\delta$ $=0.07$, Fig. $5(\mathrm{~b})]$ or be located (data not shown) in the stability field of the fcc crystal [case $\delta=0.03$, Fig. 5(a)] or in the stability field of the fluid [cases $\delta=0.12$ and 0.24 , Figs. 5(c) and 5(d)]. Also here, the re-entrant phenomenon appears as a maximum of $T$ versus $\rho$ along coexistence at densities around $\rho \approx 1.2$ at $\delta=0.07$. At the maximum, the bec and the fcc-d crystals have the same equilibrium density.
The phase diagram at high temperatures and pressures also presents an interesting behavior concerning the coexistence between the bonded fcc and the orientationally disordered fcc-d phases, consistent with a previous finding for an anisotropic octahedral model. ${ }^{13}$ Comparing the two crystals, we observe that the fcc-d is stabilized by the larger translational and rotational entropy, while the fcc is stabilized by the lower energy. As Fig. 6(a) shows in the top-right part, the fcc-fcc-d transition in the studied anisotropic model shows a density gap which progressively shrinks on increasing $T$. In the case of very small $\delta$, the two coexisting phases reach the same density. However, the solid-solid transition does not turn in a second order critical point but continues as an (orientational) order-disorder transition controlled by temperature. This transition is first order since there is always an energy difference between the two phases. ${ }^{37}$

We next look into properties of the DC and bcc crystals in the region where all bonds are formed. We start by showing the low $T$ (so that no bonds are ever broken) equilibrium density at $P=0$ in Fig. 7. Both curves can be rather well approximated by the following functional form, based on the idea that the nearest neighbors distance scales linearly with the interaction range,

$$
\rho(\delta, P=0)=\frac{\rho(0,0)}{(1+\alpha \delta)^{3}},
$$

where $\rho(0,0)$ and $\alpha$ are fitting parameters. Interestingly, the best fit value is $\alpha \approx 1 / 2$, suggesting that the average distance between bonded particles is approximately given by the hard-core diameter plus half of the range. While for the bcc case, $\rho(0,0)$ coincides with $\rho_{\mathrm{bcc}}^{\max }$, in the case of the DC crystal, the extrapolated intercept provides a value $\rho(0,0)$ 


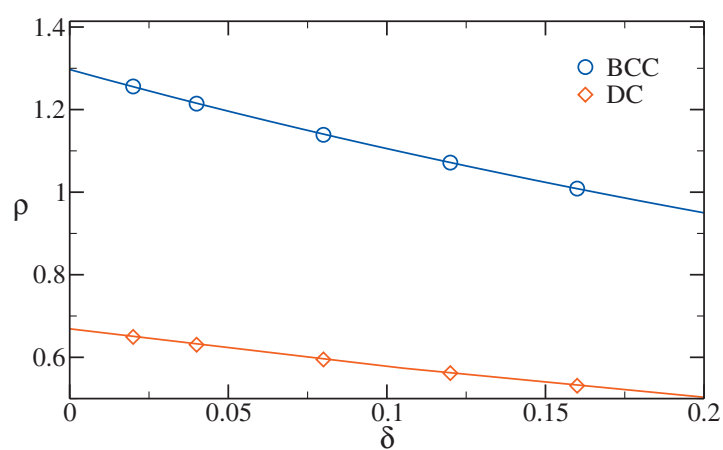

FIG. 7. Density of the bcc (circles) and DC (diamonds) as a function of $\delta$ at $P=0, T=0$. Solid lines are fit to Eq. (22). The fitting parameters for bcc are $\rho(0,0)=1.297, \alpha=0.55$, while for the DC they are $\rho(0,0)=0.669, \alpha=0.50$.

$=0.669$ which is significantly larger than $\rho_{\mathrm{DC}}^{\max }$. This suggests that the open structure of the DC crystal, due to the finite width of the bonding angle, can significantly deform without bond breaking.

In this class of square-well potentials, in the $T$ region where all bonds are formed, the excess Helmholtz free energy can be written as $A=-2 N u_{0}-T S$, where $S$ is only function of $\rho$ and not of $T$, measuring the volume available to the system without breaking bonds. Hence the density at zero pressure as a function of the range, $\rho(\delta, P=0)$, is $T$-independent, provided that $T$ is small enough so that bonds are not broken. To get a deeper insight into the properties of the crystal structures studied, we computed their entropy at low temperature as a function of the density. This was done by computing the entropy at zero pressure and $T=0.1$ as described in Sec. III B, and then by performing thermodynamic integration along the $T=0.1$ isotherm. Figure 8 (a) shows the entropy of the DC and bcc crystals in the fully bonded configurations, at different densities, for several values of the range. Since the energy of the crystal does not change with density (in the range of densities in which bonding can be achieved), the maximum of $S(\rho)$ selects the equilibrium density at zero pressure. The dependence of the zeropressure entropy on the range is shown in Fig. 8(b). The figure also shows the two contributions to the total entropy, the orientational and the translational one, which have been separately evaluated as described in Sec. III B.

The rotational contribution to the entropy is constant with $\delta$ and identical in the two bonded crystals, suggesting that the angular space explored in these two crystals is the same. The translational contribution instead increases on decreasing the number of neighbors, which progressively constrains the amplitude of the rattling motion in the bonded configuration. Interestingly enough, the slope of $S$ versus $\ln (\delta)$ for the DC case is significantly different from the one observed in the isotropic square-well fcc and in the bcc case. While in the last two cases the explored volume increases with the cube of $\delta$, in the DC case a quadratic dependence describes the data much more accurately, suggesting that the open environment around a bonded particle significantly alters the geometry of the explored volume compared to the more dense case created by the presence of a large number of neighbors, either partially (as in the bcc case) or fully bonded (as in the square-well fcc case). Figure 8(b) also reports the
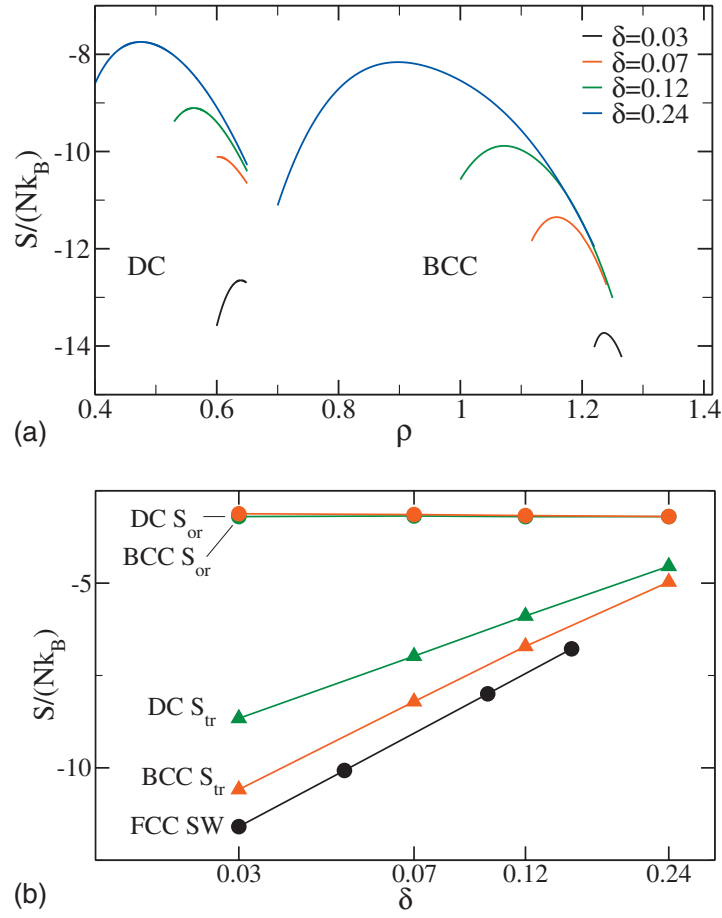

FIG. 8. (a) Configurational entropy per particle as a function of the density in the DC and bcc crystals, in the fully bonded configuration. Note that the entropies are negative since we are investigating a classical system and the kinetic contribution is missing. (b) Entropy of the crystal at zero pressure as a function of the interaction range for the DC, bcc and isotropic square-well fcc. Except in the case of the isotropic square-well fcc, where there are no orientational degrees of freedom, the translational and orientational entropy contributions are separately reported. Please note the log scale on the $x$-axis on panel (b).

entropy of the square-well potential in the fcc configuration as a reference. As expected, in this case, the dependence of $S$ on $\ln \delta$ is well described by a slope of three, $S /\left(N k_{\mathrm{B}}\right)$ $=3 \ln (0.7 \delta)$, in close agreement with the theoretical proposition by Sear. ${ }^{38}$ We also note that in the case of the hardsphere fcc, for which analytic calculations of the free volume in the spirit of the cell theory are available, ${ }^{39}$ a similar dependence on the particle-particle distance is found.

\section{CONCLUSIONS}

This manuscript reports a detailed numerical study of the phase diagram of a model with a hard-core repulsion and four tetrahedrally arranged interaction sites, focusing on the role of the interaction range on the relative stability of the different phases in the equilibrium phase diagram. The phase diagrams obtained show that, at low pressures and temperatures, the low-density diamond cubic is the stable crystal. As pressure increases, the bcc crystal becomes the most stable phase. Both the DC and bcc crystals are favored by the symmetry of the patch arrangement. At very high pressures packing effects dominate and a fully bonded fcc phase appears, even though the angle between bonded neighbors is not tetrahedral. Finally, at high temperatures, the only stable crystal is the plastic fcc structure. Interestingly, for small ranges, the fcc and fcc-d can coexist in a narrow window of densities, which shrinks on increasing $T$. 
As previously found in spherical models, ${ }^{40-42}$ the gasliquid critical points becomes metastable on decreasing the range below $\approx 0.15 \sigma$, with respect to the DC phase. For smaller values the Kern-Frenkel tetrahedral model does not have a thermodynamically stable liquid phase. But, differently from the spherical case, the metastability gap does not increase significantly on decreasing the range. ${ }^{15}$

We have observed re-entrant behavior in two coexistence curves: the fluid-DC and the bcc-fcc-d. The re-entrant behavior is observed in the equilibrium phase diagram only for specific values of the interaction range, but can nonetheless be observed as a metastable extension of the coexistence line.

We have also reported a study of the crystal properties in the fully bonded region, where the Helmholtz free energy changes only for entropic contributions. The open nature of the DC signals itself in a rather large value of the crystal entropy and in a non trivial dependence of the accessible free volume with $\delta$.

In the present study, the bonding angle has been kept constant and the role of the interaction range has been explored. This has been made possible by the use of the KernFrenkel potential, in which bond angle and bond range are completely decoupled. We plan to investigate the role of the angle on the phase diagram in a future publication. A full understanding of the role of the range and of the angle will help clarifying the conditions for optimal crystallization for particles with tetrahedral geometry.

\section{ACKNOWLEDGMENTS}

We thank G. Foffi and C. Vega for interesting discussions. We acknowledge support from NoE SoftComp NMP3-CT-2004-502235, ERC-226207PATCHYCOLLOIDS, and ITN-COMPLOIDS. E.S. is financed by a Marie Curie I-E fellowship. F.R. thanks the HPC-Europa2 program for supporting his visit to Edinburgh.

${ }^{1}$ J. Kolafa and I. Nezbeda, Mol. Phys. 61, 161 (1987).

${ }^{2}$ C. Vega and P. A. Monson, J. Chem. Phys. 109, 9938 (1998).

${ }^{3}$ A. B. Pawar and I. Kretzschmar, Macromol. Rapid Commun. 31, 150 (2010).

${ }^{4}$ S. C. Glotzer and M. J. Solomon, Nature Mater. 6, 557 (2007).

${ }^{5}$ A. Hynninen, J. H. J. Thijssen, E. C. M. Vermolen, M. Dijkstra, and A. van Blaaderen, Nature Mater. 6, 202 (2007).

${ }^{6}$ A. Lomakin, N. Asherie, and G. B. Benedek, Proc. Natl. Acad. Sci. U.S.A. 96, 9465 (1999).
${ }^{7}$ H. Liu, S. K. Kumar, and F. Sciortino, J. Chem. Phys. 127, 084902 (2007)

${ }^{8}$ C. Gögelein, G. Nägele, R. Tuinier, T. Gibaud, A. Stradner, and P. Schurtenberger, J. Chem. Phys. 129, 085102 (2008).

${ }^{9}$ E. Zaccarelli, S. V. Buldyrev, E. La Nave, A. J. Moreno, I. Saika-Voivod, F. Sciortino, and P. Tartaglia, Phys. Rev. Lett. 94, 218301 (2005).

${ }^{10}$ E. Bianchi, J. Largo, P. Tartaglia, E. Zaccarelli, and F. Sciortino, Phys. Rev. Lett. 97, 168301 (2006).

${ }^{11}$ M. G. Noro and D. Frenkel, J. Chem. Phys. 113, 2941 (2000).

${ }^{12}$ G. Foffi and F. Sciortino, J. Phys. Chem. B 111, 9702 (2007).

${ }^{13}$ E. G. Noya and C. Vega, J. Chem. Phys. 127, 054501 (2007).

${ }^{14}$ J. Chang, A. M. Lenhoff, and S. I. Sandler, J. Chem. Phys. 120, 3003 (2004).

${ }^{15}$ F. Romano, E. Sanz, and F. Sciortino, J. Phys. Chem. B 113, 15133 (2009).

${ }^{16}$ Z. Zhang, A. S. Keys, T. Chen, and S. C. Glotzer, Langmuir 21, 11547 (2005).

${ }^{17}$ L. M. Ghiringhelli, C. Valeriani, E. J. Meijer, and D. Frenkel, Phys. Rev. Lett. 99, 055702 (2007).

${ }^{18}$ I. Saika-Voivod, P. H. Poole, and R. K. Bowles, J. Chem. Phys. 124, 224709 (2006).

${ }^{19}$ T. Li, D. Donadio, and G. Galli, J. Chem. Phys. 131, 224519 (2009).

${ }^{20}$ N. Kern and D. Frenkel, J. Chem. Phys. 118, 9882 (2003).

${ }^{21}$ C. Vega, E. Sanz, J. L. F. Abascal, and E. G. Noya, J. Phys.: Condens. Matter 20, 153101 (2008).

${ }^{22}$ M. S. Wertheim, J. Stat. Phys. 35, 19 (1984).

${ }^{23}$ M. S. Wertheim, J. Stat. Phys. 35, 35 (1984).

${ }^{24}$ F. Sciortino, E. Bianchi, J. F. Douglas, and P. Tartaglia, J. Chem. Phys. 126, 194903 (2007).

${ }^{25}$ E. Bianchi, P. Tartaglia, and F. Sciortino, J. Chem. Phys. 129, 224904 (2008).

${ }^{26}$ H. Liu, S. K. Kumar, F. Sciortino, and G. T. Evans, J. Chem. Phys. 130, 044902 (2009).

${ }^{27}$ D. Frenkel and A. J. C. Ladd, J. Chem. Phys. 81, 3188 (1984).

${ }^{28}$ D. Frenkel and B. Smith, Understanding Molecular Simulations, 2nd ed. (Academic, New York, 2001).

${ }^{29}$ A. Z. Panagiotopoulos, Mol. Phys. 61, 813 (1987).

${ }^{30}$ D. A. Kofke, Mol. Phys. 78, 1331 (1993).

${ }^{31}$ A. J. C. Ladd and L. Woodcock, Chem. Phys. Lett. 51, 155 (1977).

${ }^{32}$ S. J. Singer and R. Mumaugh, J. Chem. Phys. 93, 1278 (1990).

${ }^{33}$ J. L. F. Abascal, E. Sanz, R. G. Fernández, and C. Vega, J. Chem. Phys. 122, 234511 (2005).

${ }^{34}$ B. H. Bransden and C. J. Joachain, Physics of Atoms and Molecules, 2nd ed. (Prentice-Hall, Harlow, 2003).

${ }^{35} \mathrm{D}$. Eisenberg and D. Kauzmann, The Structure and Properties of Water (Oxford University Press, New York, 1969).

${ }^{36}$ E. Sanz, C. Vega, J. L. F. Abascal, and L. G. MacDowell, Phys. Rev. Lett. 92, 255701 (2004).

${ }^{37}$ J. L. F. Abascal, C. Vega, C. McBride, and F. Bresme, Phys. Rev. E 68, 052501 (2003).

${ }^{38}$ R. P. Sear, Mol. Phys. 96, 1013 (1999).

${ }^{39}$ R. J. Buehler, J. R. H. Wentorf, J. O. Hirschfelder, and C. F. Curtiss, J. Chem. Phys. 19, 61 (1951).

${ }^{40}$ M. H. J. Hagen and D. Frenkel, J. Chem. Phys. 101, 4093 (1994).

${ }^{41}$ D. L. Pagan and J. D. Gunton, J. Chem. Phys. 122, 184515 (2005).

${ }^{42}$ H. Liu, S. Garde, and S. Kumar, J. Chem. Phys. 123, 174505 (2005). 